\title{
Lidil
}

Revue de linguistique et de didactique des langues

$54 \mid 2016$

La phrase en production d'écrits, approches nouvelles en didactique

\section{Ann-Birte Krüger, Nathalie Thamin et Stella Cambrone-Lasnes (dir.), Diversité linguistique et culturelle à l'école : accueil des élèves et formation des acteurs}

Revue Carnets d'atelier de sociolinguistique, nº 11, L'Harmattan, 2016, $288 \mathrm{p}$.

Marielle Rispail

\section{(2) OpenEdition}

Journals

Édition électronique

URL : http://journals.openedition.org/lidil/4135

DOI : 10.4000/lidil.4135

ISSN : 1960-6052

Éditeur

UGA Éditions/Université Grenoble Alpes

Édition imprimée

Date de publication : 20 novembre 2016

Pagination : 211-212

ISBN : 978-2-84310-339-1

ISSN : $1146-6480$

Référence électronique

Marielle Rispail, «Ann-Birte Krüger, Nathalie Thamin et Stella Cambrone-Lasnes (dir.), Diversité linguistique et culturelle à l'école : accueil des élèves et formation des acteurs », Lidil [En ligne], 54 | 2016, mis en ligne le 01 janvier 2017, consulté le 31 octobre 2020. URL : http://journals.openedition.org/lidil/ 4135 ; DOI : https://doi.org/10.4000/lidil.4135 
ait fulgurance. Les institutions françaises en sont un bon exemple. Un numéro supplémentaire à ce sujet viendrait compléter ces analyses très enrichissantes pour la sociolinguistique française.

Nolwenn Bailly

LIDILEM, Université Grenoble Alpes

Ann-Birte Krüger, Nathalie Thamin et Stella Cambrone-Lasnes (dir.), Diversité linguistique et culturelle à l'école : accueil des élèves et formation des acteurs, revue Carnets d'atelier de sociolinguistique, $\mathrm{n}^{\circ}$ 11, L'Harmattan, 2016, 288 p.

On attendait avec impatience ce travail collectif de nos collègues de Besançon, dont une équipe, en tissage scientifique et éthique avec des équipes du LIDILEM et d'autres partenaires (CASNAV, etc.) et réseaux, travaille avec patience et méthode sur la question de la diversité culturelle et linguistique sociale, dans son rapport avec l'école.

Cet ouvrage vient à point pour répondre à notre curiosité scientifique et aux besoins du terrain enseignant - dynamiques qui se rejoignent trop rarement. Il fait suite à plusieurs actions de formation et de réflexion fécondes autour des langues dans la classe, liant avec bonheur dans une même dynamique (cf. le sous-titre) accueil des élèves et formation des enseignants - même si ces deux volets sont séparés dans l'ouvrage, ce qui n'était peut-être pas indispensable. En effet, l'introduction, fouillée et décidée en faveur d'une «école inclusive», donne résolument le ton en posant la formation des enseignants à l'accueil des enfants de la migration comme une urgence sociale : il s'agit de «dépasser l'habitus et l'idéologie monolingues» pour penser «l'altérité au sein de l'espace scolaire», d'optimiser les apprentissages communs, tout en valorisant les langues et cultures d'origine des élèves. Les auteures veulent croire à un contexte institutionnel favorable (p. 16) pour développer une formation à la diversité, du côté des élèves comme du côté des enseignants.

On apprécie particulièrement que soient détaillés des espaces et publics souvent marginalisés dans les recherches en didactique : les classes de maternelle (Cécile Goï, Rita Carol et coll., Isabelle Audras et Françoise Leclaire) ou le périscolaire (Stella Cambrone-Lannes) par exemple, les parents dans l'école (Isabelle Audras et Françoise Leclaire), les enfants sourds (Saskia Munier) par exemple. 
La deuxième partie sur la formation des enseignants insiste, une fois de plus, sur la nécessité de travailler d'abord sur les représentations (Isabelle Lamimi), entre autres par le travail biographique réflexif (Diana-Lee Simon) maintenant assez répandu, sur les activités métalinguistiques (Lucile Cagnon et coll.), qui permettent de faire le pont entre les différentes langues en contact dans la classe, et rappelle à bon escient que la liberté pédagogique (Maryse Adam-Maillet) permet des innovations et audaces dont les enseignant-e-s ne profitent pas toujours.

Soulignons enfin deux volontés transversales qui irriguent les deux parties : celle d'éclairer la réalité française par ce qui se passe ailleurs, au Canada (Saskia Munier), au Luxembourg (Roberto Gomez-Fernandez), au Liban (Jacqueline Billiez et Carla Serhan), et celle de mettre en valeur la responsabilité des ESPE dans ce contexte français (Virginie Lapique, Andrea Young et Latisha Mary), institution qui peine parfois, au-delà des discours théoriques, à didactiser de façon concrète la prise en compte de la diversité et à donner aux enseignant-e-s qu'elle forme des outils efficaces de travail dans leurs classe, en faveur d'un plurilinguisme assumé et valorisé.

Car on est frappé, à la lecture de l'ouvrage, par le nombre de propositions pratiques qu'on peut y recenser. Merci aux auteur-e-s de nous aider, chercheur-e-s, enseignant-e-s et formateur-e-s, à mettre des actes sur des idées qui, depuis l'Éveil aux langues (Stella Cambrone-Lasnes), ne se sont peut-être pas beaucoup assez attachées à s'enraciner de façon rigoureuse et contrôlée dans les classes.

On perçoit dans cet ouvrage, dont la pluralité des signatures renforce l'unité du propos, combien est pertinente la dernière phrase d'un texte récent de Philippe Blanchet ${ }^{1}$ à l'adresse des professeurs de langues : «La diversité linguistique nous invite à développer une éthique générale de la diversité.» C'est en effet tout un projet social qui se dessine, au-delà de l'accueil des élèves allophones dans nos classes : un projet qui mettrait au centre l'altérité, les différences et leurs rencontres, dont l'école nous aurait appris les bienfaits cognitifs et le bonheur partagé.

Marielle Rispail

CELEC, Université Jean Monnet - Saint-Étienne, Université de Lyon

1. Notions trompeuses et discriminations dans l'enseignement-apprentissage des langues aux adultes, septembre 2016. 